\title{
Percus-Yevick theory for the structural properties of the seven-dimensional hard-sphere fluid
}

\author{
Miguel Robles* and Mariano López de Hard丹 \\ Centro de Investigación en Energía, UNAM, Temixco, Morelos 62580, Mexico \\ Andrés Santo: \\ Departamento de Fúsica, Universidad de Extremadura, E-06071 Badajoz, Spain
}

(Dated: October 13, 2018)

\begin{abstract}
The direct correlation function and the (static) structure factor for a seven-dimensional hardsphere fluid are considered. Analytical results for these quantities are derived within the PercusYevick theory
\end{abstract}

Interest in studying hard-core fluids in different dimensionalities is tied to the notion that, since these systems share some characteristic features such as the existence of a freezing transition, by considering a higher dimension [in which the mathematics may sometimes become simpler (see for instance Ref. 1)] one may gain insight into common phenomenology which is either untractable or rather difficult in two or three dimensions. Therefore it is not surprising that many studies have been devoted to hard-core fluids in dimensions higher than three, including fairly recent ones, mostly involving virial coefficients and/or equations of state (for a non exhaustive but hopefully representative list see Refs. $2,3,3,4,5,6)$. On the other hand, the importance of the Percus-Yevick (PY) theory ${ }^{7}$ for the structural and thermodynamic properties of liquids rests on the fact that it is exactly solvable in the case of hard-core systems in odd dimensions. $\stackrel{2.3}{=} \mathrm{In}$ fact, the direct correlation function $c(r)$ and the static structure factor $S(q)$ have been derived analytically in one, three, and five dimensions (see Ref. 3 and references therein). The major aim of this Note is to provide the explicit expressions for the functions $c(r)$ and $S(q)$ of the 7D hard-sphere fluid in the PY theory. This work extends and complements our previous paper ${ }^{\underline{5}}$ in which we concentrated on the thermodynamic properties and the virial coefficients of the same system, also within the PY approximation, and on the comparison of some of these results with our own simulation data. It must be emphasized that the highest dimensionality for which completely analytical results may be derived from the PY theory is precisely $d=7$. Beyond that dimensionality, numerical work is required at one stage or another.

Following Ref. 3, the direct correlation function of the 7D hard-sphere fluid has, in the PY theory, the polynomial form

$$
c(r)=\left(c_{0}+c_{1} r+c_{3} r^{3}+c_{5} r^{5}+c_{7} r^{7}\right) \Theta(1-r),
$$

where the diameter of a sphere is $\sigma=1$ and $\Theta(x)$ is Heaviside's step function. The coefficients $c_{i}(i=0,1,3,5,7)$ depend on the packing fraction $\eta=\left(\pi^{3} / 840\right) \rho$ (where $\rho$ is the number density). After some algebra, one finds the following structure:

$$
c_{i}=\frac{1}{(1-\eta)^{4}} \sum_{j=0}^{4} t_{i j}\left[Q^{(0)}\right]^{j} .
$$

Here, $Q^{(0)}$ is the physical solution of a quartic equation,,$\frac{5}{5}$ which reads

$$
Q^{(0)}=\frac{1}{3360 \eta(1-\eta)}\left(3-10 \eta-T_{1}^{1 / 2}-T_{2}^{1 / 2}\right),
$$

where

$$
\begin{gathered}
T_{1}=\frac{2}{3}\left(3+36 \eta+10 \eta^{2}\right)+\frac{1}{6} B \\
T_{2}=2 T_{1}-\frac{1}{2} B-\left(3-114 \eta-222 \eta^{2}-10 \eta^{3}\right) T_{1}^{-1 / 2} \\
B=A^{1 / 3}+\left(3+36 \eta+10 \eta^{2}\right)^{2} A^{-1 / 3} \\
A=-A_{1}+\sqrt{A_{1}^{2}-\left(3+36 \eta+10 \eta^{2}\right)^{6}} \\
A_{1}=459+43740 \eta-4644 \eta^{2}+16524 \eta^{3}-357948 \eta^{4} \\
+160920 \eta^{5}+23300 \eta^{6} .
\end{gathered}
$$

The coefficients $t_{i j}$ appearing in Eq. (22) are listed in Table II It can be checked that $c\left(1^{-}\right)=-g\left(1^{+}\right)$, as expected from the continuity of the indirect correlation function $\gamma(r) \equiv g(r)-c(r)-1$. As an illustration, the top panel of Fig. 1 shows $c(r)$ for $\eta=0.01,0.02, \ldots, 0.07$.

Now we turn to the structure factor $S(q)$. According to the Ornstein-Zernike equation, $S(q)$ is related to the Fourier transform $\widetilde{c}(q)$ of $c(r)$ by

$$
S(q)=\frac{1}{1-\rho \widetilde{c}(q)} .
$$

In $7 \mathrm{D}$, the Fourier transform is ${ }^{3}$

$$
\widetilde{c}(q)=(2 \pi)^{7 / 2} q^{-5 / 2} \int_{0}^{\infty} d r r^{7 / 2} J_{5 / 2}(q r) c(r),
$$


TABLE I: Expressions for the coefficients $t_{i j}$.

\begin{tabular}{|c|c|}
\hline$j$ & $t_{0 j}$ \\
\hline 0 & $-(1+6 \eta)^{2}$ \\
\hline 1 & $2688 \eta(2+5 \eta)(1+6 \eta)$ \\
\hline 2 & $-1053696 \eta^{2}\left(9+50 \eta+60 \eta^{2}\right)$ \\
\hline 3 & $1011548160 \eta^{3}(3+4 \eta)(2+5 \eta)$ \\
\hline 4 & $-141616742400 \eta^{4}(3+4 \eta)^{2}$ \\
\hline$j$ & $t_{1 j}$ \\
\hline 0 & $\frac{35}{16} \eta(4+3 \eta)^{2}$ \\
\hline 1 & $-840 \eta(4+3 \eta)\left(1+33 \eta+15 \eta^{2}\right)$ \\
\hline 2 & $6720 \eta\left(12+1212 \eta+18783 \eta^{2}+17060 \eta^{3}+3750 \eta^{4}\right)$ \\
\hline 3 & $-45158400 \eta^{2}\left(3+36 \eta+10 \eta^{2}\right)\left(1+33 \eta+15 \eta^{2}\right)$ \\
\hline 4 & $6322176000 \eta^{3}\left(3+36 \eta+10 \eta^{2}\right)^{2}$ \\
\hline$j$ & $t_{3 j}$ \\
\hline 0 & $-\frac{35}{16}(20-13 \eta)\left(12-38 \eta+33 \eta^{2}\right)$ \\
\hline 1 & $-420\left(60-2759 \eta+9163 \eta^{2}-10975 \eta^{3}+3825 \eta^{4}\right)$ \\
\hline 2 & $3360 \eta\left(22041-308370 \eta+812905 \eta^{2}-882160 \eta^{3}+273950 \eta^{4}\right)$ \\
\hline 3 & $-22579200 \eta^{2}\left(3147-19924 \eta+38685 \eta^{2}-36360 \eta^{3}+9650 \eta^{4}\right)$ \\
\hline 4 & $6322176000 \eta^{3}\left(3144-12576 \eta+17541 \eta^{2}-13360 \eta^{3}+2850 \eta^{4}\right)$ \\
\hline$j$ & $t_{5 j}$ \\
\hline 0 & $\frac{21}{16}\left(1320-5107 \eta+6282 \eta^{2}-2446 \eta^{3}\right)$ \\
\hline 1 & $252\left(330-15179 \eta+51509 \eta^{2}-59291 \eta^{3}+21945 \eta^{4}\right)$ \\
\hline 2 & $-14112 \eta\left(17325-242482 \eta+661205 \eta^{2}-674260 \eta^{3}+226550 \eta^{4}\right)$ \\
\hline 3 & $94832640 \eta^{2}\left(2475-15664 \eta+31953 \eta^{2}-27500 \eta^{3}+8050 \eta^{4}\right)$ \\
\hline 4 & $-26553139200 \eta^{3}\left(2475-9888 \eta+14702 \eta^{2}-10032 \eta^{3}+2400 \eta^{4}\right)$ \\
\hline$j$ & $t_{7 j}$ \\
\hline 0 & $-\frac{1}{16}\left(20592-79789 \eta+97872 \eta^{2}-38430 \eta^{3}\right)$ \\
\hline 1 & $-24\left(2574-118404 \eta+402761 \eta^{2}-461341 \eta^{3}+172695 \eta^{4}\right)$ \\
\hline 2 & $47040 \eta\left(3861-54054 \eta+147942 \eta^{2}-149642 \eta^{3}+51060 \eta^{4}\right)$ \\
\hline 3 & $-45158400 \eta^{2}\left(3861-24453 \eta+50151 \eta^{2}-42632 \eta^{3}+12730 \eta^{4}\right)$ \\
\hline 4 & $6322176000 \eta^{3}\left(7722-30888 \eta+46269 \eta^{2}-31056 \eta^{3}+7610 \eta^{4}\right)$ \\
\hline
\end{tabular}

TABLE II: Expressions for the functions $\mathcal{C}_{i}(q)$.

\begin{tabular}{lc}
\hline \hline$i$ & $\mathcal{C}_{i}(q)$ \\
\hline 0 & $q\left(q^{2}-15\right)$ \\
1 & $q^{4}-24 q^{2}+48$ \\
3 & $q^{6}-48 q^{4}+576 q^{2}-1152$ \\
5 & $q^{8}-80 q^{6}+2400 q^{4}-28800 q^{2}+57600$ \\
7 & $q^{10}-120 q^{8}+6720 q^{6}-201600 q^{4}+2419200 q^{2}-4838400$ \\
\hline \hline
\end{tabular}

where $J_{5 / 2}(z)=\sqrt{2 / \pi} z^{-5 / 2}\left[\left(z^{2}-3\right) \sin z-3 z \cos z\right]$ is a Bessel function of the first kind. Inserting Eq. (1) into Eq. (10) and performing the integrals one gets

$\widetilde{c}(q)=c_{0} \psi_{0}(q)+c_{1} \psi_{1}(q)+c_{3} \psi_{3}(q)+c_{5} \psi_{5}(q)+c_{7} \psi_{7}(q)$,

where

$$
\psi_{i}(q)=16 \pi^{3} q^{-(7+i)}\left[\mathcal{C}_{i}(q) \cos q-\mathcal{S}_{i}(q) \sin q-\mathcal{C}_{i}(0)\right] .
$$

The functions $\mathcal{C}_{i}(q)$ are listed in TableI, while the functions $\mathcal{S}_{i}(q)$ are

$$
\mathcal{S}_{i}(q)=\frac{d}{d q} \mathcal{C}_{i}(q)+3 q^{2+i}
$$

Insertion of Eq. (11) into Eq. (9) gives the structure factor. This function is plotted in the bottom panel of Fig. 1.
In view of the rather satisfactory agreement that has been observed between the analytical PY results for the compressibility factor of the 7D hard-sphere fluid and the simulation data, $\stackrel{5,6}{,}$ one wonders whether a similar
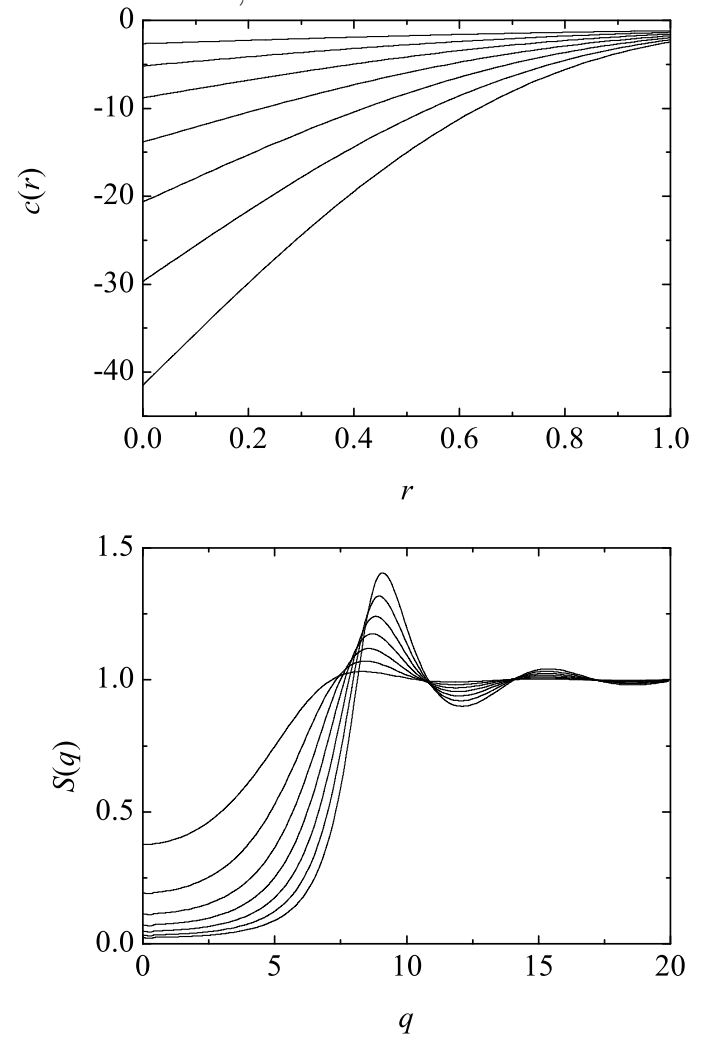

FIG. 1: Plot of the direct correlation function $c(r)$ (top panel) and of the structure factor $S(q)$ (bottom panel) of the 7D fluid of hard hyperspheres in the PY theory. The packing fractions corresponding to the curves are, from top to bottom in the left end, $\eta=0.01,0.02,0.03,0.04,0.05,0.06$, and 0.07 .

agreement will hold for the structural properties. That this is indeed the case has been recently confirmed by Bishop and his coworkers, $\frac{8}{-}$ who have found a very good agreement between their simulation results and the ones computed using our analytical formulae.

\section{Acknowledgments}

This work has been partially supported by DGAPAUNAM under project IN-110406. One of the authors (A.S.) acknowledges the financial support of Ministerio de Educación y Ciencia (Spain) through Grant No. FIS2004-01399 (partially financed by FEDER funds).

\footnotetext{
* mrp@cie.unam.mx $\quad+$ malopez@servidor.unam.mx
} 
$\ddagger$ andres@unex.es; http://www.unex.es/fisteor/andres/

1 H. L. Frisch and J. K. Percus, Phys. Rev. E 60, 2942 (1999); G. Parisi and F. Slanina, ibid. 62, 6554 (2000).

2 C. Freasier and D. J. Isbister, Mol. Phys. 42, 927 (1981).

3 E. Leutheusser, Physica A 127, 667 (1984).

${ }^{4}$ M. Luban and A. Baram, J. Chem. Phys. 763233 (1982); J. P. J. Michels and N. J. Trappeniers, Phys. Lett. A 104, 425 (1984); H. L. Frisch, N. Rivier and D. Wyler, Phys. Rev. Lett. 54, 2061 (1985); M. Baus and J. L. Colot, Phys. Rev. A 36, 3912 (1987); Y. Song, E. A. Mason, and R. M. Stratt, J. Phys. Chem. 93, 6916 (1989); J. Amorós, J. R. Solana, and E. Villar, Phys. Chem. Liq. 19, 119 (1989); M. Luban and J. P. J. Michels, Phys. Rev. A 41, 6796 (1990); D. J. González, L. E. González, and M. Silbert, Mol. Phys. 74, 613 (1991); M. J. Maeso, J. R. Solana, J. Amorós, and E. Villar, Mater. Chem. Phys. 19, 119 (1989); M. Bishop, A. Masters, and J. H. R. Clarke, J. Chem. Phys. 110, 11449 (1999); A. Santos, ibid. 112, 10680 (2000); S. B. Yuste, A. Santos, and M. López de Haro, Europhys. Lett. 52, 158 (2000); M. González-Melchor, J. Alejandre, and M. López de Haro, J. Chem. Phys. 114, 4905 (2001); M. Bishop, A. Masters, and A. Yu. Vlasov, ibid. 121, 6884 (2004); N. Clisby and B. M. McCoy, J. Stat. Phys. 114, 1343 (2004); ibid. 114, 1361 (2004); ibid. 122, 15 (2006); Pramana 64, 775 (2005); I. Lyberg, J. Stat. Phys. 119, 747 (2005); L. Lue, J. Chem. Phys. 122, 044513 (2005); M. Bishop, A. Masters, and A. Yu. Vlasov, ibid. 122, 154502 (2005); M. Bishop, P. A. Whitlock, and D. Klein, ibid. 122, 074508 (2005); M. Bishop and P. A. Whitlock, ibid. 123, 014507 (2005); A. Santos and M. López de Haro, Phys. Rev. E 72, 01050(R) (2005).

5 M. Robles, M. López de Haro, and A. Santos, J. Chem. Phys. 120, 9113 (2004); Erratum: 125 (2006).

${ }^{6}$ L. Lue and M. Bishop, Phys. Rev. E 74, 021201 (2006).

7 J. K. Percus and G. J. Yevick, Phys. Rev. 110, 1 (1958); M. S. Wertheim, Phys. Rev. Lett. 10, 321 (1963); J. Math. Phys. 5, 643 (1964); E. Thiele, J. Chem. Phys. 39, 474 (1963).

8 M. Bishop, private communication. 